\title{
The Possibility of Coherentism and the Stringency of Ceteris Paribus Conditions
}

\author{
Jonah N. SCHUPBACH
}

Received: $12 / 06 / 14$

Final Version: 26/11/14

BIBLID 0495-4548(2015)30:1p.43-52

DOI: $10.1387 /$ theoria. 11725

ABSTRACT: In (Schupbach 2008), I put forward a "possibility result" for Bayesian Coherentism, showing that there exist plausible sets of ceteris paribus conditions that imply that coherence is truth-conducive. Against this result, Schubert (2012) argues that the specific ceteris paribus conditions I consider are "jointly inconsistent". In this article, I prove to the contrary that these conditions can consistently be enforced while allowing degrees of coherence to vary. Next, I consider a related criticism, inspired by Olsson's (2005) constraints on ceteris paribus conditions. This leads to a general discussion of the principle(s) that should guide one in choosing appropriately stringent ceteris paribus conditions.

Keywords: Bayesianism, Ceteris Paribus Conditions, Coherence, Confirmation, Impossibility Results, Probability.

RESUMEN: En (Schupbach 2008) planteo un "resultado de posibilidad” para el bayesianismo coherentista mostrando que existen conjuntos plausibles de condiciones ceteris paribus que implican que la coherencia conduce a la verdad. Schubert (2012) argumenta en contra de este resultado que las condiciones ceteris paribus que considero son "inconsistentes tomadas conjuntamente". En este artículo demuestro, sin embargo, que estas condiciones pueden ser satisfechas permitiendo que los grados de coherencia varíen. Abordo después una crítica relacionada con la anterior e inspirada en los requisitos impuestos por Olsson (2005) a las condiciones ceteris paribus. Eso lleva a una discusión general del principio, o principios, que debería guiarnos para elegir unas condiciones ceteris paribus que sean apropiadas, es decir, que no sean ni muy restrictivas, ni demasiado laxas.

Palabras clave: bayesianismo, condiciones ceteris paribus, coherencia, confirmación, resultado de imposibilidad, probabilidad.

\section{Introduction}

Bovens and Hartmann's $(2003 ; 2006)$ “impossibility result” for Bayesian Coherentism asserts that "there cannot exist a measure of coherence that is probabilistic and induces a coherence ordering [...] and that simultaneously makes it the case that the more coherent the information set, the more confident we are that the information is true, ceteris paribus" (2003, 21). In a previous article (Schupbach 2008), I point out that this result is sensitive to choice of ceteris paribus conditions; Bovens and Hartmann specify a particular set of ceteris paribus conditions, and this move is crucial to the proof of their impossibility result. Moreover, I note that choice of coherence measure intersects with choice of ceteris paribus conditions; the ceteris paribus conditions that seem appropriate to endorse will vary depending on how one explicates the notion of coherence.

In order to clarify exactly why this is a problem for Bovens and Hartmann, I offer a "possibility result" for Bayesian Coherentism. I assume, for the sake of the argument, that 
one accepts Shogenji's (1999) measure of coherence. For any $n$-membered information set $\left\{A_{1}, A_{2}, \ldots, A_{n}\right\}$, this measure is defined as follows:

$$
C\left(\left\{A_{1}, A_{2}, \ldots, A_{n}\right\}\right)={ }_{\text {def }} \frac{\operatorname{Pr}\left(A_{1} \wedge A_{2} \wedge \ldots \wedge A_{n}\right)}{\operatorname{Pr}\left(A_{1}\right) \times \operatorname{Pr}\left(A_{2}\right) \times \ldots \times \operatorname{Pr}\left(A_{n}\right)}
$$

The specific ceteris paribus conditions for coherence that Bovens and Hartmann adopt make little sense given the Shogenji measure. Accordingly, I suggest the following alternative set of conditions (where $E_{i}$ represents the proposition that witness $i$ asserts that $A_{i}$ is true): ${ }^{1}$

(1) Total individual strength $\operatorname{Pr}\left(A_{1}\right) \times \operatorname{Pr}\left(A_{2}\right) \times \ldots \times \operatorname{Pr}\left(A_{n}\right)$ should be held fixed.

(2) Expectedness of the reports $\operatorname{Pr}\left(E_{1} \wedge E_{2} \wedge \ldots \wedge E_{n}\right)$ should be held fixed.

(3) Reliability of the information sources should be held fixed.

Condition (3) is formally vague; however, minimally, I propose that (3) should at least entail that the true positive rate $\operatorname{Pr}\left(E_{i} \mid A_{i}\right)$ be held fixed for all $i_{.}^{2}$

Given the Shogenji measure and this alternative set of ceteris paribus conditions, it is easy to show that coherence is truth-conducive in the relevant sense: the more coherent the information set, the more confident we ought to be that the information is true, ceteris paribus. But it is also true that Shogenji's account provides a measure of coherence that is probabilistic and induces a coherence ordering. The upshot is that there exist compelling probabilistic accounts of coherence and corresponding sets of ceteris paribus conditions that do not fall prey to Bovens and Hartmann's impossibility result; Bayesian Coherentism is possible after all.

This paper considers two objections to this defense of Bayesian Coherentism's possibility, both of which criticize ceteris paribus conditions (1)-(3) as overly stringent. After arguing against both objections, I offer some general remarks about the stringency of ceteris paribus conditions and the principles that correctly guide one in choosing such conditions.

\section{Do (1)-(3) Fix Coherence?}

Schubert $(2012,7)$ argues that I have "not managed to defeat the impossibility result." His critique rests on the claim that conditions (1)-(3) entail a constant degree of coherence (as

${ }^{1}$ Note that measure $C$ makes no reference at all to any $E_{i}$; that is, the Shogenji measure applies to information sets apart from any witness context. Nonetheless, Bovens and Hartmann frame their entire study of coherence within such a context, including their impossibility result. Consequently, one must introduce ceteris paribus conditions pertaining to such a witness context in order to investigate their bearing on Bovens and Hartmann's impossibility result. For more on coherence and witness scenarios, see (Olsson 2005, ch. 2).

2 Actually, the requirement is weaker than this. For the possibility result to go through, it merely needs to be the case that there exists some plausible explication of reliability, which entails that if we hold reliability fixed, then the true positive rate be held fixed (but not necessarily that if we hold the true positive rate fixed, we are thereby holding reliability fixed). In (2008, 329), I motivate this formal requirement by showing that Bovens and Hartmann seem to require this as part of their own reliability condition. 
measured by $C$ ). That is, one cannot vary degree of coherence while enforcing these ceteris paribus conditions. If this is true, it does indeed spell trouble for the possibility result. It amounts to showing that the ceteris paribus conditions driving that result are not plausibly describing "all else" other than coherence but instead encompass coherence as well. In this case, one cannot possibly have differing degrees of coherence ceteris paribus, and so one cannot possibly show that coherence is truth-conducive in the relevant sense.

Stated formally, Schubert's central claim is as follows (where we are comparing two probability distributions $P r$ and $P r^{\prime}$ and where $n=2$ ):

Schubert's Claim. If all of the following conditions hold:

(1) $\operatorname{Pr}\left(A_{1}\right) \times \operatorname{Pr}\left(A_{2}\right)=\operatorname{Pr}^{\prime}\left(A_{1}\right) \times \operatorname{Pr}^{\prime}\left(A_{2}\right)$

(2) $\operatorname{Pr}\left(E_{1} \wedge E_{2}\right)=\operatorname{Pr}^{\prime}\left(E_{1} \wedge E_{2}\right)$

(3) $\operatorname{Pr}\left(E_{1} \mid A_{1}\right)=\operatorname{Pr}^{\prime}\left(E_{1} \mid A_{1}\right), \operatorname{Pr}\left(E_{2} \mid A_{2}\right)=\operatorname{Pr}^{\prime}\left(E_{2} \mid A_{2}\right), \operatorname{Pr}\left(E_{1} \mid \neg A_{1}\right)=\operatorname{Pr}^{\prime}\left(E_{1} \mid \neg A_{1}\right)$, and $\operatorname{Pr}\left(E_{2} \mid \neg A_{2}\right)=\operatorname{Pr}^{\prime}\left(E_{2} \mid \neg A_{2}\right), 3$

then it necessarily follows that:

$$
C_{P r}\left(\left\{A_{1}, A_{2}\right\}\right)=\frac{\operatorname{Pr}\left(A_{1} \wedge A_{2}\right)}{\operatorname{Pr}\left(A_{1}\right) \times \operatorname{Pr}\left(A_{2}\right)}=\frac{\operatorname{Pr}^{\prime}\left(A_{1} \wedge A_{2}\right)}{\operatorname{Pr}^{\prime}\left(A_{1}\right) \times \operatorname{Pr}^{\prime}\left(A_{2}\right)}=C_{P r^{\prime}}\left(\left\{A_{1}, A_{2}\right\}\right) .
$$

Because the terms in the denominators of $C_{P r}\left(\left\{A_{1}, A_{2}\right\}\right)$ and $C_{P r^{\prime}}\left(\left\{A_{1}, A_{2}\right\}\right)$ must be equal by condition (1), this amounts to the claim that (1)-(3) imply that $\operatorname{Pr}\left(A_{1} \wedge A_{2}\right)=\operatorname{Pr}^{\prime}\left(A_{1} \wedge A_{2}\right)$.

In order to prove this claim, Schubert investigates a particular probability distribution according to which $\operatorname{Pr}\left(A_{1}\right)=\operatorname{Pr}\left(A_{2}\right)=.5, \operatorname{Pr}\left(A_{1} \wedge A_{2}\right)=.25, \operatorname{Pr}\left(E_{1} \mid A_{1}\right)=\operatorname{Pr}\left(E_{2} \mid A_{2}\right)=.75$, and $\operatorname{Pr}\left(E_{1} \mid \neg A_{1}\right)=\operatorname{Pr}\left(E_{2} \mid \neg A_{2}\right)=.25$. From these probabilities, one can calculate $C_{P r}\left(\left\{A_{1}, A_{2}\right\}\right)=1$ and $\operatorname{Pr}\left(E_{1} \wedge E_{2}\right)=.25$. Schubert's strategy then is to instantiate the contrapositive of his claim by showing that another distribution $P r^{\prime}$ that results in a differing amount of coherence (so that $\left.\operatorname{Pr}\left(A_{1} \wedge A_{2}\right) \neq \operatorname{Pr}^{\prime}\left(A_{1} \wedge A_{2}\right)\right)$ will have to break with at least one of conditions (1)-(3). To this end, Schubert stipulates that $\operatorname{Pr}^{\prime}\left(A_{1} \wedge A_{2}\right)=.375 \neq \operatorname{Pr}\left(A_{1} \wedge A_{2}\right)$. Through a clever bit of algebra, Schubert shows that this stipulated fact and condition $(3)$ together imply the following system of equations:

3 Note the discrepancy between my condition (3) and Schubert's strengthening of this condition. Interestingly, while the possibility result only requires the weaker version of (3), Schubert's proposed proof (as summarized below) relies crucially upon the stronger condition - which additionally requires that $\operatorname{Pr}\left(E_{i} \mid \neg A_{i}\right)$ be held fixed, for all $i$. To see this, notice that specific values for $\operatorname{Pr}^{\prime}\left(A_{1} \wedge \neg A_{2}\right)$, $\operatorname{Pr}^{\prime}\left(\neg A_{1} \wedge A_{2}\right)$, and $\operatorname{Pr}^{\prime}\left(\neg A_{1} \wedge \neg A_{2}\right)$ are no longer determined by equations (E1) and (E2) when the values of $\operatorname{Pr}^{\prime}\left(E_{1} \mid \neg A_{1}\right)$ and $\operatorname{Pr}^{\prime}\left(E_{2} \mid \neg A_{2}\right)$ are unknown. But then values for $\operatorname{Pr}^{\prime}\left(A_{1}\right)$ and $\operatorname{Pr}^{\prime}\left(A_{2}\right)$ are likewise undetermined, and thus the argument no longer shows that ceteris paribus condition (1) is broken. One could therefore argue that Schubert's argument misses its mark, since it really criticizes a stronger set of conditions than those required for the possibility result. Here, however, we will take a different tack in criticizing Schubert's argument. 


$$
\begin{aligned}
\operatorname{Pr}^{\prime} & \left(A_{1} \wedge \neg A_{2}\right) \operatorname{Pr}^{\prime}\left(E_{1} \mid A_{1}\right) \operatorname{Pr}^{\prime}\left(E_{2} \mid \neg A_{2}\right) \\
& +\operatorname{Pr}^{\prime}\left(\neg A_{1} \wedge A_{2}\right) \operatorname{Pr}^{\prime}\left(E_{1} \mid \neg A_{1}\right) \operatorname{Pr}^{\prime}\left(E_{2} \mid A_{2}\right) \\
& +\operatorname{Pr}^{\prime}\left(\neg A_{1} \wedge \neg A_{2}\right) \operatorname{Pr}^{\prime}\left(E_{1} \mid \neg A_{1}\right) \operatorname{Pr}^{\prime}\left(E_{2} \mid \neg A_{2}\right)=5 / 128
\end{aligned}
$$

$$
\operatorname{Pr}^{\prime}\left(A_{1} \wedge \neg A_{2}\right)+\operatorname{Pr}^{\prime}\left(\neg A_{1} \wedge A_{2}\right)+\operatorname{Pr}^{\prime}\left(\neg A_{1} \wedge \neg A_{2}\right)=5 / 8
$$

By enforcing conditions (2) and (3), Schubert is able to solve these equations, proving in this case that $\operatorname{Pr}^{\prime}\left(A_{1} \wedge \neg A_{2}\right)=\operatorname{Pr}^{\prime}\left(\neg A_{1} \wedge A_{2}\right)=0$ and $\operatorname{Pr}^{\prime}\left(\neg A_{1} \wedge \neg A_{2}\right)=5 / 8$. Given these values, we have $\operatorname{Pr}^{\prime}\left(A_{1}\right)=\operatorname{Pr}^{\prime}\left(A_{1} \wedge A_{2}\right)+\operatorname{Pr}^{\prime}\left(A_{1} \wedge \neg A_{2}\right)=.375$ and $\operatorname{Pr}^{\prime}\left(A_{2}\right)=\operatorname{Pr}^{\prime}\left(A_{1} \wedge A_{2}\right)+\operatorname{Pr}^{\prime}\left(\neg A_{1} \wedge A_{2}\right)=.375$. From this it follows that $\operatorname{Pr}\left(A_{1}\right) \times \operatorname{Pr}\left(A_{2}\right)=1 / 4 \neq 9 / 64=\operatorname{Pr}^{\prime}\left(A_{1}\right) \times \operatorname{Pr}^{\prime}\left(A_{2}\right)$, and so condition (1) is not satisfied. In Schubert's words (p. 9): “[T] his contradicts Schupbach's clause (1) [...] At least one of Schupbach's ceteris paribus-clauses must be given up. Hence his argument against Bovens and Hartmann's impossibility proof, which relies on all three clauses, does not hold."

In order to evaluate Schubert's criticism, first recall his central claim that conditions (1)-(3) entail fixed degrees of coherence, as measured by $C$. Schubert's putative proof of this general point explores in detail a single case, a particular pair of probability distributions in which degrees of coherence are well-defined and differ in a particular way. But this is an odd strategy to take when the goal is to prove a general claim about all pairs of probability distributions that satisfy (1)-(3). Effectively, Schubert's "proof" attempts to deduce a universal conclusion on the basis of one particular instance; he attempts to argue that All pairs of probability distributions that entail $C_{P r}\left(\left\{A_{1}, A_{2}\right\}\right) \neq C_{P r^{\prime}}\left(\left\{A_{1}, A_{2}\right\}\right)$ necessarily must break with one of the ceteris paribus conditions (1), (2), or (3) by proving that There exist pairs of probability distributions (at least one) that entail that $C_{P r}\left(\left\{A_{1}, A_{2}\right\}\right) \neq C_{P r^{\prime}}\left(\left\{A_{1}, A_{2}\right\}\right)$ but that break with one of these conditions.

In proving this latter point, Schubert is effectively showing that not all cases in which coherence varies will be cases in which (1)-(3) can be enforced; in other words, allowable differences in coherence between probability distributions are constrained by the enforcement of ceteris paribus conditions. However, it is the very nature of ceteris paribus conditions to constrain one's choice of available comparisons. Thus, it should come as no surprise that there exist specific cases rendered incomparable by (1)-(3). We cannot just pick whatever probability distributions we like and expect that they will be comparable ceteris paribus. It seems to me that this is the legitimate conclusion of Schubert's argument.

Still, one might fairly wonder whether Schubert is right that conditions (1)-(3) force coherence to be held constant. That is, perhaps Schubert's conclusion is correct even if his proof is misguided. The question is whether coherence can possibly fluctuate even while one enforces conditions (1), (2), and (3). It is not difficult to find distributions that show that it can. In fact, one can take Schubert's own example but recommend a different fluctuation in coherence (i.e., different than Schubert's proposed fluctuation) without running into any conflict with the ceteris paribus conditions.

Instead of increasing $\operatorname{Pr}\left(A_{1} \wedge A_{2}\right)=.25$ to $\operatorname{Pr}^{\prime}\left(A_{1} \wedge A_{2}\right)=.375$ (a move which Schubert shows is not allowed by the ceteris paribus conditions), we can decrease the same term (e.g., to $\left.\operatorname{Pr}^{\prime}\left(A_{1} \wedge A_{2}\right)=.17\right)$ without breaking with any of conditions (1)-(3) - see Proof 1 in appendix A. Moreover, whereas in this example we are comparing cases in which $A_{1}$ and $A_{2}$ 
are statistically independent and negatively relevant respectively, there are also such cases in which the propositions in question are positively relevant - see Proof 2 in appendix A. Indeed, contra Schubert, there are any number of such examples that can be put forward wherein degree of coherence can vary under conditions (1)-(3).

\section{Complete Separability as a Guide to Holding All Else Equal}

At the heart of Schubert's criticism is the idea that (1)-(3) are too stringent. We have shown above that, contra Schubert, these conditions do not imply fixed degrees of coherence. However, one might still argue that these conditions are too strong. Doing so requires spelling out plausible necessary constraints on what it is appropriate to include in a set of ceteris paribus conditions. If (1), (2), or (3) break any such constraints, than these are still too strong in the sense that the culpable condition $(s)$ is barring certain comparisons that ought to be considered.

Olsson $(2002 ; 2005)$ proposes such a constraint. According to him, in order to "isolate a target property" (e.g., coherence) by enforcing ceteris paribus conditions, we must only be allowed to fix properties that are "completely separable" from the target property in the sense that changes to the former properties do not at all affect the latter property. ${ }^{4}$ So, for example, he objects even to holding the size of an information set constant in $C$ s seteris paribus conditions given that $C$ "is sensitive to" this number $(2005,123)$.

If one accepts this as the appropriate criterion for choosing ceteris paribus conditions, then conditions (1)-(3) are indeed too stringent and consequently the possibility result is ill-founded. For one thing, the size of an information set is assumed equal across sets in my proof of the possibility result. In addition, as Olsson notes, condition (1) in particular would require us to fix values of an information set's total individual strength $\operatorname{Pr}\left(A_{1}\right) \times \operatorname{Pr}\left(A_{2}\right) \times \ldots \times \operatorname{Pr}\left(A_{n}\right)$. However, this same term is the denominator of $C$. Consequently, changing an information set's total individual strength would manifestly affect its degree of coherence; fixing strength via our ceteris paribus conditions would "impose limitations on the extent to which the degree of coherence can consistently vary" $(2005,122)$.

Of course, this point will only cast the possibility result into doubt to the extent that Olsson's criterion proves plausible. In defense of this criterion, Olsson asserts that violations of it would intuitively imply lack of full independent variability between factors (2005, 118); but there is no prior argument for the idea that ceteris paribus conditions must only fix factors that are fully independently variable with respect to the target property. In general, Olsson's discussion of, and case for, this criterion is driven by the idea that,

${ }^{4}$ Olsson first requires that appropriately fixed properties "vary independently" of the target property, where this requirement is violated in case "fixing the value of the one property imposes limitations on the extent to which the other property can consistently vary" $(2005,118)$. However, he effectively weakens this requirement to that stated above in his discussion and examples. For example, he argues that the size of information sets should not be held constant by coherence's ceteris paribus conditions given that changes to an information set's size (adding or subtracting members) "typically affects the degree of coherence," in spite of the fact that changes in size per se do not limit degree of coherence's range. When dealing with properties that are mathematically explicated, Olsson suggests that complete separability is lost when the target property can be represented as a function of another property. 
when aiming to prove a positive thesis about the isolated influence of coherence, one ought to fix as little as possible. Heeding this advice safeguards one's study from concluding that something is generally true when really it is only true of an artificially restricted set of cases. But there is another danger from which such a study needs safeguarding, the very danger that motivates the enforcement of ceteris paribus conditions in the first place: that one might fail to isolate the target property and so allow other factors to confound our study. To avoid this danger, one must be sure to fix enough factors.

The problem with Olsson's criterion is that it focuses solely on avoiding the first danger, leaving his account susceptible to the second. That is, this principle apparently does not allow one to fix enough. One can point to cases where, in order to isolate some property, we would clearly want to fix some particular factor - else our study would be confounded - despite the fact that this factor is not completely separable from the target property. To take one example, say we are interested in investigating whether dietary habits have an influence on lung cancer rates. It is understood that whether or not someone is a smoker influences their dietary habits. Thus, if we should not hold factors constant that affect the factor of interest, then in this case, we would not want to control for smoking when trying to isolate the influence of diet on lung cancer rates. But this would clearly be to allow smoking to confound our study. In this case, even though smoking and dietary habits are not "completely separable" from one another, it is still apparent that we should control for smoking when testing the influence of diet on lung cancer. In the same way, it may well be that the coherence of an information set is sensitive to that set's total individual strength $\operatorname{Pr}\left(A_{1}\right) \times \operatorname{Pr}\left(A_{2}\right) \times \ldots \times \operatorname{Pr}\left(A_{n}\right)$, but that we should still control for the latter in order to isolate the influence of the former.

\section{Regarding the Stringency of Ceteris Paribus Conditions}

We have now discussed two objections to conditions (1)-(3). And I have argued against both, but for different reasons. On the one hand, Schubert's argument assumes a necessary constraint on ceteris paribus conditions, one that is so obviously correct that it hardly requires mentioning: in order to hold all else equal, one should only fix factors other than the target property. Let us call this principle partial separability, the idea being that a property must at least be partially separable from the target property in order to be appropriately fixed by the latter's ceteris paribus conditions. Schubert argues that (1)-(3) collectively break this condition, that these jointly imply that we're fixing coherence itself. But while partial separability is surely a sound constraint on one's choice of ceteris paribus conditions, I have shown that Schubert is mistaken; (1)-(3) fix properties that are partially separable from coherence.

On the other hand, there is nothing wrong with the argument showing that these same properties are not completely separable from coherence. The second objection asserts that since this is the case, these properties have no place in coherence's ceteris paribus conditions. But the problem here is with the principle of complete separability that it relies on; viz., that in order to hold all else equal, one should only fix factors completely separable from the target property. I have argued that this principle does not allow us to fix all of the appropriate properties; it prohibits us from controlling for confounding factors. 
Should we require partial separability alone of our ceteris paribus conditions? In this case, we would require that any factor be held constant (in addition to those already fixed), so long as doing so does not also fix the target property. ${ }^{5}$ This proposal runs into at least two important problems. First, the criterion would underdetermine the sets of factors that ought to be fixed as part of a target property's ceteris paribus conditions. Consider again coherence explicated using $C$. Recall that $C$ s denominator explicates a notion of "total individual strength" that is conceptually distinct from coherence. But $C$ s numerator also explicates a conceptually distinct notion, namely, the joint plausibility of the relevant information prior to receiving any reports of that information. Partial separability, taken as the necessary and sufficient criterion for determining ceteris paribus conditions, forbids us from fixing total individual strength and plausibility simultaneously in order to isolate $C$. However, it requires that we fix one or other of these factors individually. The problem is that it provides us with conflicting recommendations regarding which to fix. Considering strength first, we should hold this factor constant; from thereon, the principle of partial separability will ban us from fixing joint plausibility. Considering joint plausibility first, we should fix this property; thereafter, we will not be allowed to hold strength constant lest we also fix $C$. So we receive conflicting advice depending on the order in which we consider various factors.

Second, like complete separability, this criterion would lead to counterintuitive results. Specifically, this principle falls prey to the first sort of danger mentioned above; it apparently requires one to fix too much. One can point to cases where, in order to isolate some property, we would clearly not want to fix some particular factor - else our study would be artificially constrained - despite the fact that doing so would not thereby fix our target property. Say we are interested in investigating the effects of depression on body weight. While diet surely has an influence on depression, one's diet does not determine whether he or she will be depressed; it is partially separable from this target property. But then, according to this criterion, we should fix diet when isolating the effects of depression on body weight. To do so, however, would seem tantamount to delimiting our study artificially; after all, diet is arguably an important factor through which depression might affect body weight.

Both of these problems indicate that the principle of partial separability is too lenient. It is too easy for factors to satisfy this requirement and thus we end up holding fixed too many things. In order to keep our ceteris paribus analyses from becoming artificially constrained, we will often need to let certain factors vary, despite the fact that holding them steady would not fix our target property.

5 A more defensible statement of this candidate principle would have to include a further caveat. When isolating some target property, we are typically interested in uncovering whether that property alone has an influence on a particular "end property"; in the examples above, we want to know whether coherence alone is conducive to confirmation or whether dietary habits alone have an influence on lung cancer rates. Taking partial separability as the criterion dividing factors that should from those that should not be fixed, we would be required to fix values of end properties in standard cases where these do not determine our target properties; lung cancer rates do not determine people's dietary habits, and so partial separability would have us fix lung cancer rates in order to isolate the effects of diet on lung cancer rates! A more defensible principle of partial separability would thus have us fix all properties other than end properties that are partially separable from our target property. 
The upshot is that, in order to isolate the influence of a target property, we clearly must require appropriately fixed properties to be partially separable from this property. However, this requirement taken by itself is too weak; a strictly stronger principle is needed. By contrast, we open our investigation to confounding influences if we go so far as to require that factors be completely separable; this requirement is too strong. So the correct principle(s) for choosing appropriate ceteris paribus conditions must lie somewhere between partial and complete separability. Ultimately, more work is required to uncover the elusive principle(s), but the goal is worthy. Amongst other things, an accurate evaluation of conditions (1)-(3) and further investigation into the alleged impossibility of Bayesian Coherentism wait on such an account.

\section{A. Changing Values of C While Enforcing (1)-(3)}

The point of this appendix is to prove that it is possible to have changing degrees of coherence (as measured by $C$ ) even while enforcing conditions (1)-(3). Following Schubert's lead, we focus on scenarios in which $n=2$. Accordingly, we wish to prove the following:

Changing Coherence Ceteris Paribus. There exist cases in which all of the following conditions hold:

(1) $\operatorname{Pr}\left(A_{1}\right) \times \operatorname{Pr}\left(A_{2}\right)=\operatorname{Pr}^{\prime}\left(A_{1}\right) \times \operatorname{Pr}^{\prime}\left(A_{2}\right)$

(2) $\operatorname{Pr}\left(E_{1} \wedge E_{2}\right)=\operatorname{Pr}^{\prime}\left(E_{1} \wedge E_{2}\right)$

(3) $\operatorname{Pr}\left(E_{1} \mid A_{1}\right)=\operatorname{Pr}^{\prime}\left(E_{1} \mid A_{1}\right), \operatorname{Pr}\left(E_{2} \mid A_{2}\right)=\operatorname{Pr}^{\prime}\left(E_{2} \mid A_{2}\right), \operatorname{Pr}\left(E_{1} \mid \neg A_{1}\right)=\operatorname{Pr}^{\prime}\left(E_{1} \mid \neg A_{1}\right)$, and $\operatorname{Pr}\left(E_{2} \mid \neg A_{2}\right)=\operatorname{Pr}^{\prime}\left(E_{2} \mid \neg A_{2}\right)$,

and for which it is also true that:

$$
C_{P r}\left(\left\{A_{1}, A_{2}\right\}\right)=\frac{\operatorname{Pr}\left(A_{1} \wedge A_{2}\right)}{\operatorname{Pr}\left(A_{1}\right) \times \operatorname{Pr}\left(A_{2}\right)} \neq \frac{\operatorname{Pr}^{\prime}\left(A_{1} \wedge A_{2}\right)}{\operatorname{Pr}^{\prime}\left(A_{1}\right) \times \operatorname{Pr}^{\prime}\left(A_{2}\right)}=C_{\operatorname{Pr}^{\prime}}\left(\left\{A_{1}, A_{2}\right\}\right) .
$$

Because the denominators of $C_{P r}\left(\left\{A_{1}, A_{2}\right\}\right)$ and $C_{P r^{\prime}}\left(\left\{A_{1}, A_{2}\right\}\right)$ must be equal by (1), it suffices to show that there exist cases in which (1)-(3) are satisfied and $\operatorname{Pr}\left(\neg A_{1} \wedge A_{2}\right) \neq \operatorname{Pr}^{\prime}\left(\neg A_{1} \wedge A_{2}\right)$.

Notation. In what follows, let $x=\operatorname{Pr}^{\prime}\left(A_{1} \wedge \neg A_{2}\right), y=\operatorname{Pr}^{\prime}\left(\neg A_{1} \wedge A_{2}\right)$, and $z=\operatorname{Pr}^{\prime}\left(\neg A_{1} \wedge \neg A_{2}\right)$.

Proof 1. Begin with probability distribution $\operatorname{Pr}$, which includes all of the following information: $\operatorname{Pr}\left(A_{1}\right)=\operatorname{Pr}\left(A_{2}\right)=.5, \operatorname{Pr}\left(A_{1} \wedge A_{2}\right)=.25, \operatorname{Pr}\left(E_{1} \mid A_{1}\right)=\operatorname{Pr}\left(E_{2} \mid A_{2}\right)=.75$ , and $\operatorname{Pr}\left(E_{1} \mid \neg A_{1}\right)=\operatorname{Pr}\left(E_{2} \mid \neg A_{2}\right)=.25$. As Schubert shows, from these probabilities, one can calculate $C_{P r}\left(\left\{A_{1}, A_{2}\right\}\right)=1$ and $\operatorname{Pr}\left(E_{1} \wedge E_{2}\right)=.25$.

Now we consider the case where, instead of increasing $\operatorname{Pr}\left(A_{1} \wedge A_{2}\right)=.25$ to $\operatorname{Pr}^{\prime}\left(A_{1} \wedge A_{2}\right)=.375$ (a move which Schubert shows is not allowed by the ceteris paribus conditions), we decrease the same term to $\operatorname{Pr}^{\prime}\left(A_{1} \wedge A_{2}\right)=.17$. Schubert $(2012,8)$ shows that the following equality holds in any witness context (as defined by Bovens and Hartmann): 


$$
\begin{aligned}
\operatorname{Pr}^{\prime}\left(E_{1} \wedge E_{2}\right)= & \operatorname{Pr}^{\prime}\left(A_{1} \wedge A_{2}\right) \operatorname{Pr}^{\prime}\left(E_{1} \mid A_{1}\right) \operatorname{Pr}^{\prime}\left(E_{2} \mid A_{2}\right) \\
& +x \operatorname{Pr}^{\prime}\left(E_{1} \mid A_{1}\right) \operatorname{Pr}^{\prime}\left(E_{2} \mid \neg A_{2}\right) \\
& +y \operatorname{Pr}^{\prime}\left(E_{1} \mid \neg A_{1}\right) \operatorname{Pr}^{\prime}\left(E_{2} \mid A_{2}\right) \\
& +z \operatorname{Pr}^{\prime}\left(E_{1} \mid \neg A_{1}\right) \operatorname{Pr}^{\prime}\left(E_{2} \mid \neg A_{2}\right)
\end{aligned}
$$

Given (3) and the stipulated fact that $\operatorname{Pr}^{\prime}\left(A_{1} \wedge A_{2}\right)=.17$, we can calculate the first summand on the right hand side of this equation:

$$
\operatorname{Pr}^{\prime}\left(A_{1} \wedge A_{2}\right) \operatorname{Pr}^{\prime}\left(E_{1} \mid A_{1}\right) \operatorname{Pr}^{\prime}\left(E_{2} \mid A_{2}\right)=.17 \times .75 \times .75=.095625 \text {. }
$$

Subtracting this value from $\operatorname{Pr}^{\prime}\left(E_{1} \wedge E_{2}\right)=.25$ (known by (2)) and filling in the values (given to us via (3)) of $\operatorname{Pr}^{\prime}\left(E_{1} \mid A_{1}\right), \operatorname{Pr}^{\prime}\left(E_{2} \mid A_{2}\right), \operatorname{Pr}^{\prime}\left(E_{1} \mid \neg A_{1}\right)$, and $\operatorname{Pr}^{\prime}\left(E_{2} \mid \neg A_{2}\right)$, we get the following:

$$
.1875 x+.1875 y+.0625 z=.154375
$$

$\operatorname{Pr}^{\prime}\left(A_{1} \wedge A_{2}\right), x, y$, and $z$ must sum to 1 ; therefore, we also get the following:

$$
x+y+z=.83
$$

Unlike the system of equations that Schubert examines ((E1) and (E2)), equations (E1') and (E2') do not suffice to determine the values of $x, y$, and $z$ (proof omitted). The question thus arises whether any solutions exist that satisfy the lone remaining condition (1) we have already enforced our other conditions above, and so we have already ensured that these are satisfied. (1) may be stated in terms of $\operatorname{Pr}^{\prime}\left(A_{1} \wedge A_{2}\right)=.17, x$, and $y$ as follows:

$$
(x+.17)(y+.17)=.25
$$

Solutions to equations (E1'), (E2'), and (E3') do exist (calculations omitted), including the following two: $\{x=.116061, y=.703939, z=.01\},\{x=.703939, y=.116061, z=.01\}$.

Proof 1 compares cases in which $A_{1}$ and $A_{2}$ are statistically independent and negatively relevant respectively. In the following alternative proof, we instead look at an instance in which these propositions are positively relevant.

Proof 2. Begin with probability distribution $\operatorname{Pr}$, which includes all of the following information: $\operatorname{Pr}\left(A_{1}\right)=.6, \operatorname{Pr}\left(A_{2}\right)=.5, \operatorname{Pr}\left(A_{1} \wedge A_{2}\right)=.4, \operatorname{Pr}\left(E_{1} \mid A_{1}\right)=.7, \operatorname{Pr}\left(E_{2} \mid A_{2}\right)=.6$, and $\operatorname{Pr}\left(E_{1} \mid \neg A_{1}\right)=3 / 8, \operatorname{Pr}\left(E_{2} \mid \neg A_{2}\right)=3 / 7$. From these probabilities, one can calculate $C_{P r}\left(\left\{A_{1}, A_{2}\right\}\right)=4 / 3$.

Now consider the case where we increase $\operatorname{Pr}\left(A_{1} \wedge A_{2}\right)=.4$ to $\operatorname{Pr}^{\prime}\left(A_{1} \wedge A_{2}\right)=.5$. Given (3), we can calculate the first summand on the right hand side of $\left(^{*}\right)$ as follows: 


$$
\operatorname{Pr}^{\prime}\left(A_{1} \wedge A_{2}\right) \operatorname{Pr}^{\prime}\left(E_{1} \mid A_{1}\right) \operatorname{Pr}^{\prime}\left(E_{2} \mid A_{2}\right)=.5 \times .7 \times .6=.21
$$

Subtracting this value from $\operatorname{Pr}^{\prime}\left(E_{1} \wedge E_{2}\right)$ (known by (2)) and filling in the values (given to us via (3)) of $\operatorname{Pr}^{\prime}\left(E_{1} \mid A_{1}\right), \operatorname{Pr}^{\prime}\left(E_{2} \mid A_{2}\right), \operatorname{Pr}^{\prime}\left(E_{1} \mid \neg A_{1}\right)$, and $\operatorname{Pr}^{\prime}\left(E_{2} \mid \neg A_{2}\right)$, we get the following:

$$
.3 x+.225 y+.1607 z=.088714
$$

$\operatorname{Pr}^{\prime}\left(A_{1} \wedge A_{2}\right), x, y$, and $z$ must sum to 1 ; therefore, we also get the following:

$$
x+y+z=.5
$$

The question now arises whether any solutions exist that satisfy the lone remaining condition (1):

$$
(x+.5)(y+.5)=.3
$$

Solutions to equations (E1"), (E2"), and (E3") do exist, including the following: $\{x=.029054, y=.0607050, z=.403896\}$.

\section{Acknowledgements}

Thanks to Lydia McGrew, Erik Olsson, William Roche, and Stefan Schubert for helpful feedback on earlier drafts of this paper. Research for this article was supported by an Aldrich Fellowship from the University of Utah's Tanner Humanities Center.

\section{REFERENCES}

Bovens, Luc and Stephan Hartmann. 2003. Bayesian Epistemology. Oxford: Oxford University Press. -. 2006. An Impossibility Result for Coherence Rankings. Philosophical Studies 128: 77-91.

Olsson, Erik J. 2002. What is the Problem of Coherence and Truth? The Journal of Philosophy 94: 246-272. -. 2005. Against Coherence: Truth, Probability, and Justification. Oxford: Oxford University Press.

Schubert, Stefan. 2012. Is Coherence Conducive to Reliability? Synthese 187: 607-621.

Schupbach, Jonah N. 2008. On the Alleged Impossibility of Bayesian Coherentism. Philosophical Studies 141 (3): 323-331.

Shogenji, Tomoji. 1999. Is Coherence Truth-Conducive? Analysis 59 (4):338-345.

Jonah N. Schupbach is Assistant Professor of Philosophy at the University of Utah. He received a Ph.D. in History and Philosophy of Science from the University of Pittsburgh in 2011. His research interests have to do with the study of human reasoning and scientific methodology. He has published articles in top journals, including The British Journal for the Philosophy of Science, Philosophical Studies, Philosophy of Science, and Synthese. For more information, see his homepage: <http://jonahschupbach.com>. 